
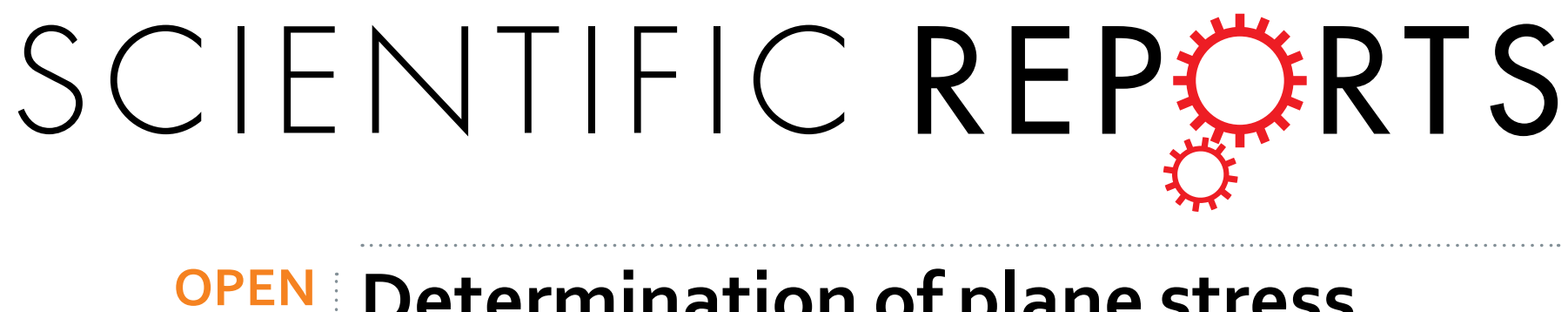

\title{
Determination of plane stress state using terahertz time-domain spectroscopy
}

Received: 22 August 2016

Accepted: 13 October 2016

Published: 08 November 2016

\author{
Zhiyong Wang ${ }^{1}$, Kai Kang ${ }^{1}$, Shibin Wang ${ }^{1}$, Lin'an $\mathrm{Li}^{1}$, Ningning $\mathrm{Xu}^{2}$, Jiaguang $\mathrm{Han}^{3}$, \\ Mingxia $\mathrm{He}^{3}$, Liang $\mathrm{Wu}^{3}$ \& Weili Zhang ${ }^{2,3}$
}

THz wave has been increasingly applied in engineering practice. One of its outstanding advantages is the penetrability through certain optically opaque materials, whose interior properties could be therefore obtained. In this report, we develop an experimental method to determine the plane stress state of optically opaque materials based on the stress-optical law using terahertz timedomain spectroscopy (THz-TDS). In this method, two polarizers are combined into the conventional THz-TDS system to sense and adjust the polarization state of THz waves and a theoretical model is established to describe the relationship between phase delay of the received $\mathrm{THz}$ wave and the plane stress applied on the specimen. Three stress parameters that represent the plane stress state are finally determined through an error function of THz wave phase-delay. Experiments were conducted on polytetrafluoroethylene (PTFE) specimen and a reasonably good agreement was found with measurement using traditional strain gauges. The presented results validate the effectiveness of the proposed method. The proposed method could be further used in nondestructive tests for a wide range of optically opaque materials.

Terahertz $(\mathrm{THz})$ wave has obtained wide applications in various fields since powerful sources were developed ${ }^{1,2}$. As a key spectral analysis technique in the $\mathrm{THz}$ waveband, $\mathrm{THz}$-TDS obtained many valuable applications in characterization of semiconductors and biological materials ${ }^{3-6}, 2 \mathrm{D}$ and tomographic imaging ${ }^{7,8}$, and geometric parameter measurement ${ }^{9}$. One outstanding feature of the $\mathrm{THz}$ wave is its high transmission ability through most optically opaque materials ${ }^{10-12}$, so that it can be used to determine and analyze the internal properties of these materials.

Stress state is important interior information of optically opaque materials as a response to external mechanical loadings. Recently, researchers reported their progresses in studying the stress-optical effect in $\mathrm{THz}$ regime ${ }^{13-17}$. In order to clarify the subtle differences among these works, a brief introduction to the stress-optical law is presented. The stress-optical law states that mechanical stress makes an originally isotropic material become optically anisotropic and the optical axes of the stress induced birefringence are aligned with the stress principle axes. If the specimen is under the plane stress state, the refractive index variations can be expressed using the stress parameters according to the following equation ${ }^{18}$

$$
\begin{aligned}
& \Delta N_{1}=A \sigma_{1}+B \sigma_{2}, \\
& \Delta N_{2}=A \sigma_{2}+B \sigma_{1} .
\end{aligned}
$$

where $\Delta N_{1}$ and $\Delta N_{2}$ are the variations in material refractive index along the directions of the principle stresses, and $A$ and $B$ are called stress-optical coefficients. For a plane stressed specimen, three parameters, including $\sigma_{1}$, $\sigma_{2}$ and $\theta$, are needed to describe its stress state. Here, $\sigma_{1}$ and $\sigma_{2}$ are the so-called principle stresses, while $\theta$ is the angle between the $x$-axis and $\sigma_{1}$. Apparently, the plane stress state can be determined if $\Delta N_{1}$ and $\Delta N_{2}$, and $\theta$ are obtained in advance. Subtract Eq. (2) from Eq. (1), it can be obtained that

${ }^{1}$ Department of Mechanics, School of Mechanical Engineering, Tianjin University, Tianjin, 300072, China. ${ }^{2}$ School of Electrical and Computer Engineering, Oklahoma State University, Stillwater, Oklahoma, 74078, USA. ${ }^{3}$ Center for Terahertz Waves and College of Precision Instrument and Optoelectronics Engineering, Tianjin University, Tianjin, 300072, China. Correspondence and requests for materials should be addressed to Z.W. (email: zywang@tju.edu.cn) orW.Z. (email: weili.zhang@okstate.edu) 


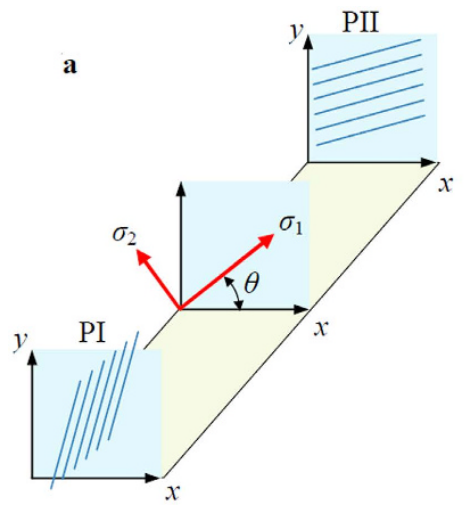

b

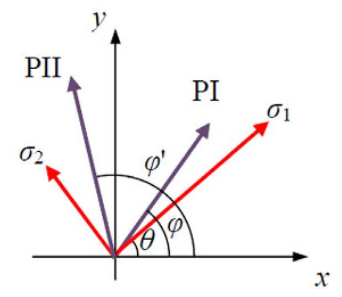

Figure 1. Orientation of the polarizers and the principle stress (a) and the projection view (b). PI: Polarizer I, PII: Polarizer II.

$$
\Delta N_{1}-\Delta N_{2}=(A-B)\left(\sigma_{1}-\sigma_{2}\right)=C\left(\sigma_{1}-\sigma_{2}\right),
$$

where parameter $C$ is the stress-optical coefficient used in the photoelasticity and $C=A-B$.

To the best knowledge of the authors, the research work on stress measurement using $\mathrm{THz}$ radiation was launched in 2004 by Tsuguhiro ${ }^{13}$. An experimental system was built based on back-ward wave oscillator to carry out experiments on a polyethylene specimen. The difference between $\Delta N_{1}$ and $\Delta N_{2}$ was measured and the stress-optic coefficient $C$ was obtained. By using a movable loading stage, the intensity distribution of the transmitted $\mathrm{THz}$ wave was also obtained when the polarizer and analyzer formed a cross-Nicol system. However, the stress information was not extracted from the intensity distribution. Ebara et al. constructed a polarized sensitive TDS system that could detect small birefringence ${ }^{14}$. Using this highly sensitive THz-TDS system, they measured the stress-caused birefringence, i.e. $\Delta N_{1}-\Delta N_{2}$, when a uniaxial stress was applied on a specimen. According to their experimental result, the stress-optic coefficient $C$ of PTFE was determined. Pfleger et al. measured the birefringence and the optical axes orientation of the $\mathrm{LiNbO}_{3} \mathrm{crystal}^{15}$. But, the presented method can not be directly used for stress measurement due to relatively small magnitude of the stress induced-birefringence. Recently, we obtained the relationship between the uniaxial tensile stress and the refractive index change $\Delta N_{1}$ and the stress-optical coefficient $A$ of PTFE $^{16,17}$.

In the above works, the stress-caused refractive index changes have been experimentally measured as $\Delta$ $N_{1}-\Delta N_{2}$ or $\Delta N_{1}$. Based the above results, it is straight forward to measure a uniaxial stress, in which there is only one stress parameter to be measured. However, it is impossible to determine a plane stress state, in which there are three unknown parameters to be determined. Although great effort was made to characterizing birefringence in $\mathrm{THz}$ regime ${ }^{19,20}$, no work succeeded on determination of the plane stress state using $\mathrm{THz}$ wave.

In this report, we develop an experimental method to determine the plane stress state in optically opaque materials using an improved THz-TDS system. An experimental principle is also established with respect to the improved THz-TDS system. The proposed method is experimentally validated and the plane stress states of PTFE specimens were obtained.

\section{Methods}

Compared with the conventional THz-TDS, the system used for plane stress state measurement is improved by integrating two polarizers to sense and adjust the polarization state of the $\mathrm{THz}$ waves. Figure 1 schematically shows the orientations of the two added polarizers and the stress components. In the improved THz-TDS system, the emitted $\mathrm{THz}$ wave is horizontally polarized. Suppose the phase and the amplitude of the emitted $\mathrm{THz}$ wave are 0 and 1 respectively, the electric field $E_{0}$ can be expressed using the Jones vector as

$$
E_{0}=\left[\begin{array}{l}
1 \\
0
\end{array}\right]
$$

As the incident $\mathrm{THz}$ radiation transmits through the specimen that is loaded by a plane stress, it is divided into two polarized radiation along the two principal stress directions, which are perpendicular to each other. As illustrated in Fig. 1, the THz wave subsequently passes Polarizer I, the specimen, and Polarizer II. The received $\mathrm{THz}$ wave can therefore be expressed as

$$
E_{1}=P_{0} \cdot Q_{\varphi}^{\prime} \cdot J_{\theta} \cdot Q_{\varphi} \cdot E_{0},
$$

where

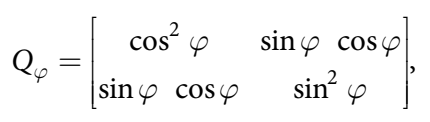




$$
\begin{gathered}
J_{\theta}=\left[\begin{array}{cc}
\cos \theta & -\sin \theta \\
\sin \theta & \cos \theta
\end{array}\right] \cdot\left[\begin{array}{cc}
\exp \left(i \delta_{1}\right) & 0 \\
0 & \exp \left(i \delta_{2}\right)
\end{array}\right] \cdot\left[\begin{array}{cc}
\cos \theta & \sin \theta \\
-\sin \theta & \cos \theta
\end{array}\right], \\
Q_{\varphi}^{\prime}=\left[\begin{array}{cc}
\cos ^{2} \varphi^{\prime} & \sin \varphi^{\prime} \cos \varphi^{\prime} \\
\sin \varphi^{\prime} \cos \varphi^{\prime} & \sin ^{2} \varphi^{\prime}
\end{array}\right],
\end{gathered}
$$

and

$$
P_{0}=\left[\begin{array}{ll}
1 & 0 \\
0 & 0
\end{array}\right]
$$

In Eq. (5), $Q_{\varphi}$ and $Q_{\varphi}^{\prime}$ are the Jones matrix of the two polarizers, $\varphi$ and $\varphi^{\prime}$ are the angles between Polarizer I, II and the horizontal direction respectively, $J_{\theta}$ is the Jones matrix of the loaded specimen, $\theta$ is the angle between the first principal stress and the horizontal direction, $\delta_{1}$ and $\delta_{2}$ are the stress-caused phase delays of the two polarized components along the directions of $\sigma_{1}$ and $\sigma_{2}$, and $P_{0}$ is used to extract the horizontally polarized component because the detector in the TDS system can only detect that component of the THz radiation. Equation (5) can be simplified as

$$
E_{1}=\left[\begin{array}{c}
T e^{i \alpha} \\
0
\end{array}\right]=\left[\begin{array}{c}
R \cos \alpha+i I \sin \alpha \\
0
\end{array}\right]
$$

where $T e^{i \alpha}$ represents the received THz wave. $R$ and $I$ are the real and the imaginary parts, and $\alpha$ is the phase delay of the received $\mathrm{THz}$ wave caused by the applied stress. After simplification, the phase delay $\alpha$ can be expressed as

$$
\tan \alpha=\frac{P \sin \delta_{1}+Q \sin \delta_{2}}{P \cos \delta_{1}+Q \cos \delta_{2}}
$$

where

$$
\begin{aligned}
& P=\cos \varphi \cdot \cos \varphi^{\prime} \cdot \cos \left(\varphi^{\prime}-\theta\right) \cdot \cos (\varphi-\theta) . \\
& Q=\cos \varphi \cdot \cos \varphi^{\prime} \cdot \sin \left(\varphi^{\prime}-\theta\right) \cdot \sin (\varphi-\theta) .
\end{aligned}
$$

Speaking specifically, the phase delays, $\delta_{1}$ and $\delta_{2}$, are caused by the stress-optical effect and the Poisson effect when stresses are loaded on the specimen and can be calculated using the following equations as

$$
\begin{aligned}
& \delta_{1}=\frac{2 \pi d f}{c}\left[\left(A-\frac{\mu\left(N_{0}-1\right)}{E}\right) \sigma_{1}+\left(B-\frac{\mu\left(N_{0}-1\right)}{E}\right) \sigma_{2}\right], \\
& \delta_{2}=\frac{2 \pi d f}{c}\left[\left(A-\frac{\mu\left(N_{0}-1\right)}{E}\right) \sigma_{2}+\left(B-\frac{\mu\left(N_{0}-1\right)}{E}\right) \sigma_{1}\right] .
\end{aligned}
$$

where $f$ is the frequency of the THz radiation, $c$ is the speed of light in air, $d$ is the original thickness of the specimen, $N_{0}, E$ and $\mu$ are the initial refractive index, the elastic modulus and the Poisson ratio of the specimen, respectively. Therefore, the relationship between the phase delay $\alpha$ of the captured $\mathrm{THz}$ wave and the applied plane stress state can be determined by combining Eqs (11, 14 and 15).

To measure the three stress parameters, the two polarizers are placed at three different orientations and the phase delay data between free and loaded conditions are captured. Since the experimentally captured THz signals are broadband, the phase delay at the $n$th frequency component $f_{n}$ for the $k$ th polarizer orientation is denoted as $\alpha_{n}^{k}$. An error function of the phase delay is thereby defined as:

$$
e\left(\sigma_{1}, \sigma_{2}, \theta\right)=\sum_{k=1}^{3} \sum_{n=1}^{N}\left[\bar{\alpha}_{n}^{k}\left(\sigma_{1}, \sigma_{2}, \theta\right)-\alpha_{n}^{k}\right]^{2},
$$

where $\bar{\alpha}_{n}^{k}\left(\sigma_{1}, \sigma_{2}, \theta\right)$ is the phase delay calculated using Eqs $\left(11,14\right.$ and 15) for any arbitrary set of $\left(\sigma_{1}, \sigma_{2}, \theta\right)$. The Newton-Raphson method is used to obtain the minimum of this error function. The specific value of $\left(\sigma_{1}, \sigma_{2}, \theta\right)$ that minimizes the error function is regarded as the real plane stress state undergone by the specimen.

Experiments. Experiments were conducted on a dumbbell-shaped PTFE sheet specimen. The specimen was fixed on a mechanical loading device that provided the uniaxial tensile loading. The phase spectra of the free and loaded specimen were measured. Meanwhile, strain gauges were glued on the specimen surface to measure the tensile strain, which was used for stress calculation for comparison. Table 1 presents the geometric and physical parameters of the PTEF specimen.

In the improved THz-TDS system, two wired-grid polarizers were added to the standard THz-TDS system, where a femtosecond laser with $88 \mathrm{MHz}$ repetition rate, $26 \mathrm{fs}$ pulse width, and $800 \mathrm{~nm}$ wavelength was used as the pump source with a $10 \mathrm{~mW}$ average power for both the THz transmitter and receiver. The THz wave was generated and detected by shorting the dipole-type photoconductive antenna gap. Because the refractive index change to be measured would be very small, high signal stability is critical for experimental measurement. In order to 


\begin{tabular}{|l|c|}
\hline Parameters & Value \\
\hline Thickness $(d)$ & $2 \mathrm{~mm}$ \\
\hline Elastic Modulus $(E)$ & $468.5 \mathrm{MPa}$ \\
\hline Poisson ratio $(\mu)$ & 0.4 \\
\hline Refractive index $\left(n_{0}\right)$ & 1.417 \\
\hline
\end{tabular}

Table 1. Physical and Geometry Parameters.
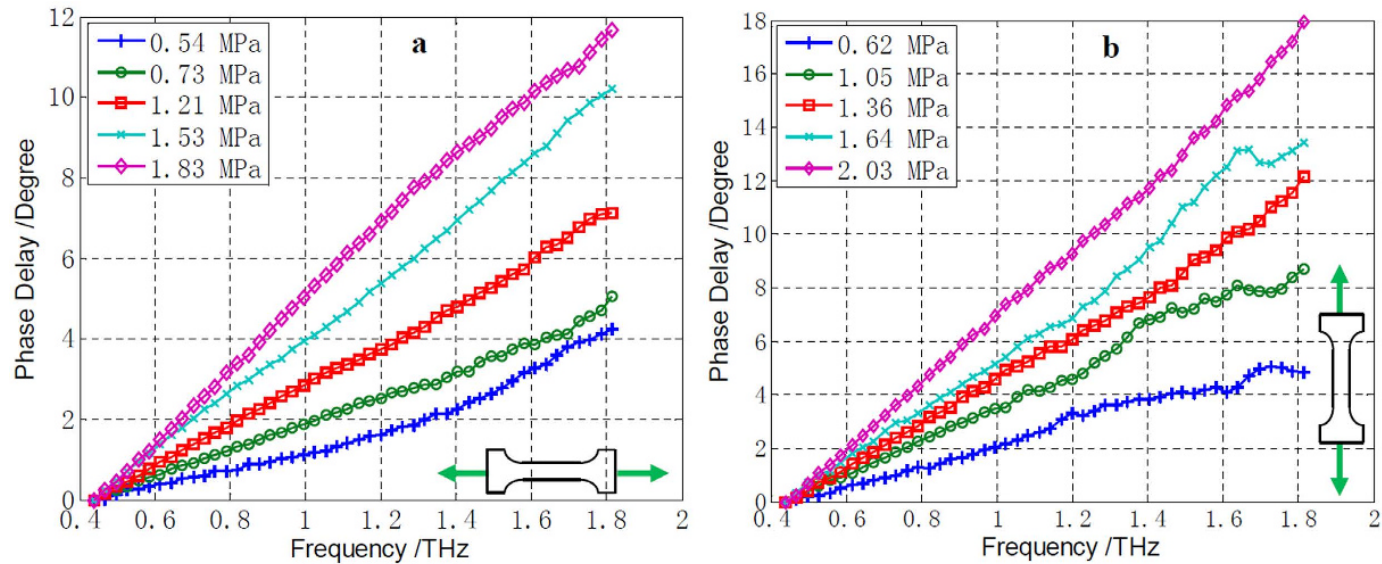

Figure 2. Phase delays under uniaxial stress. (a) Phase delays when horizontal stress loads were applied. (b) Phase delays when vertical stress loads were applied.

keep the THz signal stable, humidity is controlled below $2 \%$ by mixing dry air into the sealed chamber during the measurement. The highly reliable frequency range of the THz-TDS system is $0.4 \sim 1.80 \mathrm{THz}$.

\section{Results}

Stress-optical coefficients measurement. The two stress-optical coefficients $A$ and $B$ are imperative in stress state calculation and can only be obtained through experiments. In order to measure coefficient $A$ of the PTFE specimen, the loading device was placed horizontally so that a horizontal uniaxial stress was applied on the specimen. The polarization directions of the two polarizers were also set horizontal. In this case, $\theta, \varphi, \varphi^{\prime}$ and $\sigma_{2}$ are all equal to zero and $\sigma_{1}$ is equal to the horizontally applied horizontal stress. Thus, stress-optical coefficient $A$ can be obtained from Eqs (11-14) as

$$
A=\frac{c}{2 \pi d} \cdot \frac{\alpha}{f} \cdot \frac{1}{\sigma_{h}}+\frac{\mu N_{0}}{E},
$$

where $\sigma_{h}$ is the applied horizontal uniaxial stress and $\alpha / f$ represents the slope of phase delay with respect to frequency when a stress is applied on the specimen. Figure 2 a shows five phase delay vs. frequency curves under different horizontal stresses. The stress-optical coefficient $A$ is thus obtained as $2.03 \times 10^{-9} \mathrm{~Pa}^{-1}$ using Eq. (17).

The stress-optical coefficient $B$ was similarly obtained, except that the loading device was vertically placed to obtain a vertical stress. The two polarizers were kept horizontal. In this case, $\theta=90^{\circ}, \varphi=0, \varphi^{\prime}=0, \sigma_{2}=0$, and $\sigma_{1}$ equals to the vertically applied stress. The coefficient $B$ could be obtained from Eqs (11-13 and 15) as

$$
B=\frac{c}{2 \pi d} \cdot \frac{\alpha}{f} \cdot \frac{1}{\sigma_{v}}+\frac{\mu N_{0}}{E},
$$

where $\sigma_{v}$ is the vertically applied uniaxial stress. Figure $2 \mathrm{~b}$ shows the phase delay vs. frequency when different vertical stresses were applied. The stress-optical coefficient $B$ is then obtained as $2.63 \times 10^{-9} \mathrm{~Pa}^{-1}$ according to Eq. (18).

Plane stress state measurement. The plane stress state of the PTFE specimen was measured under four different loadings. In the first two experiments, horizontal loadings were applied on the specimen, while vertical loading were applied in the other two experiments. The applied stress states are obtained by strain gauge and listed in Table 2 for comparison. The specimen is supposed to be under general plane stress states. As stated previously, in order to obtain specimen plane stress state that is actually represented by the three stress parameters, $\sigma_{1}, \sigma_{2}$ and $\theta$, the phase delay of the captured $\mathrm{THz}$ waves needs to be measured at three different orientation settings of the two polarizers. The three orientation settings in the experiment were: 1) $\varphi=0, \varphi^{\prime}=0$;2) $\varphi=45^{\circ}, \varphi^{\prime}=45^{\circ}$; and 3) $\varphi=60^{\circ}$, $\varphi^{\prime}=-60^{\circ}$, respectively. For each orientation setting, the THz wave was captured for both free and loading conditions. The phase delays at different frequency components were obtained by Fourier transform and served as $\alpha_{n}^{k}$ in Eq. (16). 


\begin{tabular}{|l|c|c|}
\hline No. & By THz-TDS (MPa, MPa, Degree) & By strain gauge (MPa, MPa, Degree) \\
\hline 1 & $(1.7,-0.1,13)$ & $(1.7,0,0)$ \\
\hline 2 & $(2.2,-0.7,-14)$ & $(2.5,0,0)$ \\
\hline 3 & $(1.8,-0.5,79)$ & $(1.7,0,90)$ \\
\hline 4 & $(2.4,0.2,114)$ & $(2.5,0,90)$ \\
\hline
\end{tabular}

Table 2. Measurement Results of the Stress State.

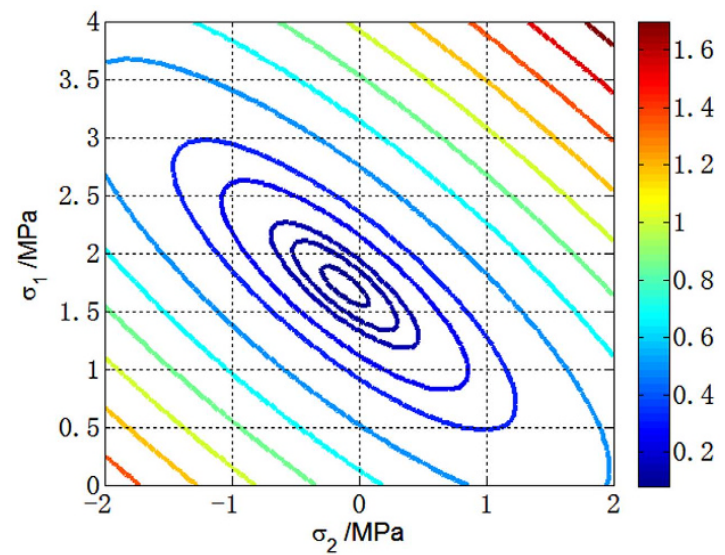

Figure 3. Distribution of the error function $e\left(\sigma_{1}, \sigma_{2}\right)$ when $\theta=13^{\circ}$.

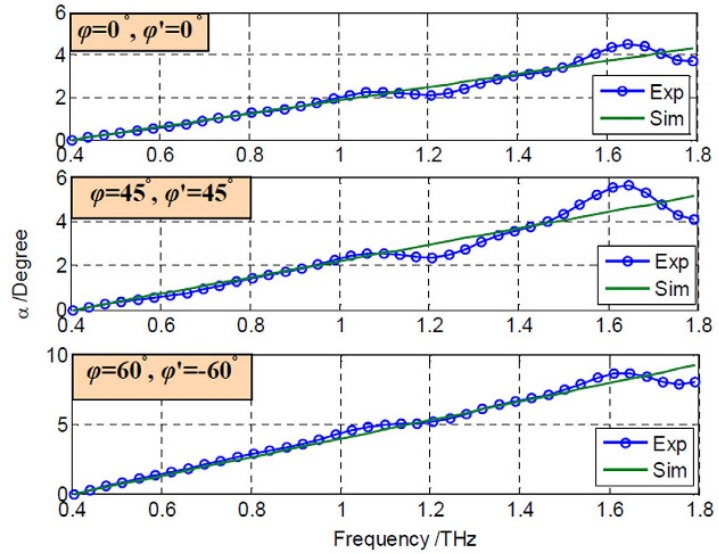

Figure 4. Phase delays of the first experimental measurement and theoretical simulation.

The measured plane stress states using the proposed $\mathrm{THz}$ method are presented in Table 2 . The stress states are represented by the stress parameters that make the error function minimum under each loading condition. For the first loading condition, the plane stress state obtained by THz-TDS is $\sigma_{1}=1.7 \mathrm{MPa}, \sigma_{2}=-0.1 \mathrm{MPa}$, and $\theta=13^{\circ}$. Figure 3 shows the distribution of the error function in the $\sigma_{1}-\sigma_{2}$ plane when $\theta=13^{\circ}$. A well-shaped minimum is shown in the figure. Thus, it is reliable to achieve the minimum by the Newton-Raphson scheme. Figure 4 compares the theoretical and experimental phase delays at the frequency domain under the first plane stress state. Good agreements are indicated between the experimental results and the theoretical calculation for the three polarizer orientation settings. The error function distributions and phase delay curves of the other three measurements are provided in Supplementary Figures.

\section{Discussion}

Comparison between the plane stress states obtained by the proposed method and the results by the strain gauges indicates a relatively apparent difference in the stress parameters $\sigma_{2}$ and $\theta$. Several factors may account for the difference. One possible reason may be the nonlinear mechanical property of PTFE. It could induce some inevitable error when strain gauges are used to measure the stress state. It is assumed in this report that free specimens are optically isotropic. But actually residual birefringence may exist in unstressed specimens. This may be another influencing factor. Stability of the THz signal also affects the precision of stress measurement. Another factor may 
be the relatively low extinction ratios of the metallic wire-grid THz polarizers used in the THz-TDS system. Thus, the penetrated $\mathrm{THz}$ waves would not be perfectly polarized. Therefore, further efforts should be contributed to improving accuracy and stability of the proposed method.

It may also be noticed that in the proposed method only the phase data of the received signal is used in stress determination, while the magnitude information is ignored, which is different or contradictory to conventional $\mathrm{THz}$ wave applications. This is because the amplitude data of our THz-TDS system is not stable enough. The instability of the amplitude data makes it impractical to achieve accurate measurement on stress states.

Using the proposed method, $\mathrm{THz}$ waves can not only be used to find interior flaw in materials, but also to determine the stress distribution when material under mechanical loading. Some flaws may be too small to be detected, but they induce obviously singular stress distributions that could be identified by stress measurement. Thus, the proposed stress measurement method here is possibly developed to be a prospective non-destructive testing method in future.

\section{References}

1. Ferguson, B. \& Zhang, X. C. Materials for terahertz science and technology. Nature Mater 1, 26-33 (2006).

2. Tonouchi, M. Cutting-edge terahertz technology. Nature Photon. 1, 97-105 (2007).

3. Tassin, P., Koschny, T., Kafesaki, M. \& Soukoulis, C. M. A comparison of graphene, superconductors and metals as conductors for metamaterials and plasmonics. Nat. Photon 6(4), 259-264 (2012).

4. Baxter, J. B. \& Schmuttenmaer, C. A. Conductivity of ZnO Nanowires, Nanoparticles, and Thin Films Using Time-Resolved Terahertz Spectroscopy. J. Phys. Chem. B 110(50), 25229-25239 (2006).

5. Koda, S., Mori, T. \& Kojima, S. Broadband terahertz dynamics of propylene glycol monomer and oligomers, J. Mol. Struct. 1126(15), 127-131 (2016).

6. Laman, N. \& Grischkowsky, D. Terahertz conductivity of thin metal films. Appl. Phys. Lett. 93(5), 051105 (2008).

7. Mittlleman, D. M., Jacobsen, R. H. \& Nuss, M. C. T-ray imaging. IEEE J. Sel. Top. Quant. Electron. 2, 679-692 (1996)

8. Mittleman, D. M. et al. Recent advances in terahertz imaging. Appl. Phys. B. 68(6), 1085-1094 (1999)

9. Ho, L. et al. Effects of film coating thickness and drug layer uniformity on in vitro drug release from sustained-release coated pellets: A case study using terahertz pulsed imaging. Int. J. Pharm. 382, 151-159 (2009).

10. Hangyo, M., Tani, M. \& Nagashima, T. Terahertz time-Domain spec-troscopy of solids: a review. J. Infrared Milli. Terahz. Waves. 26, $1661-1690$ (2005).

11. Wang, S. \& Zhang, X. C. Pulsed terahertz tomography. J. Appl. Phys. D. 37, R1-36 (2004).

12. Reid, M. \& Fedosejevs R. Terahertz birefringence and attenuation properties of wood and paper. Appl. Opt. 45, 2766-2772 (2006).

13. Tsuguhiro, T. Observation of Cavity Interface and Mechanical Stress in Opaque Material by THz Wave In Behavior of Electromagnetic Waves in Different Media and Structures (ed. Ali, A.) 383-398 (Croatia, 2011).

14. Ebara, S., Hirota, Y., Tani, M. \& Hangyo, M. Highly sensitive birefrin-gence measurement in THz frequency region and its application to stress measurement In Proc. of Joint 32nd International Conference on Infrared and Millimeter Waves and the 15th International Conference on Terahertz Electronics 666-667 (2007).

15. Pfleger, M. et al. Advanced birefringence measurements in standard terahertz time-domain spectroscopy. Appl. Opt. 53, 3183-3190 (2014).

16. Li, L. A. et al. Active modulation of refractive index by stress in the terahertz frequency range. Appl. Opt. 52, 6364-6368 (2013).

17. Song, W. \& Wang, Z. Y. Active modulation of refractive index by stress in the terahertz frequency range: erratum. Appl. Opt. 55, 2223 (2016).

18. Hetenyi, M. Handbook of experimental stress analysis. 839 (Hoboken, 1950).

19. Wiesauer, K. \& Jördens, C. Recent advances in birefringence studies at THz frequencies. J. Infrared Milli Terahz Waves. 34, 663-681 (2013).

20. Nagashima, T., Tani, M. \& Hangyo, M. Polarization-sensitive THz-TDS and its application to anisotropy aensing. J. Infrared Milli Terahz Waves. 34, 740-775 (2013).

\section{Acknowledgements}

This work was funded by the National Natural Science Foundation of China under Grant Nos 11572217 and 61205096.

\section{Author Contributions}

Conceived idea and experiments- Z.W. Performed experiments- K.K., N.X. and L.W. Prepared manuscript- Z.W. Critical reading of manuscript- S.W., L.L., J.H., M.H. and W.Z.

\section{Additional Information}

Supplementary information accompanies this paper at http://www.nature.com/srep

Competing financial interests: The authors declare no competing financial interests.

How to cite this article: Wang, Z. et al. Determination of plane stress state using terahertz time-domain spectroscopy. Sci. Rep. 6, 36308; doi: 10.1038/srep36308 (2016).

Publisher's note: Springer Nature remains neutral with regard to jurisdictional claims in published maps and institutional affiliations.

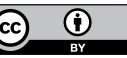

This work is licensed under a Creative Commons Attribution 4.0 International License. The images or other third party material in this article are included in the article's Creative Commons license, unless indicated otherwise in the credit line; if the material is not included under the Creative Commons license, users will need to obtain permission from the license holder to reproduce the material. To view a copy of this license, visit http://creativecommons.org/licenses/by/4.0/

(c) The Author(s) 2016 